\title{
Long-term risk of ischemic heart disease after adjuvant radiotherapy in breast cancer: results from a large population- based cohort
}

Anna-Karin Wennstig ${ }^{1,2^{*}}$, Charlotta Wadsten ${ }^{1,3}$, Hans Garmo ${ }^{4,5}$, Irma Fredriksson ${ }^{6,7}$, Carl Blomqvist ${ }^{8}$, Lars Holmberg ${ }^{5,9}$, Greger Nilsson ${ }^{10,11,12}$ and Malin Sund ${ }^{1}$

\begin{abstract}
Background: Adjuvant radiotherapy (RT) for breast cancer (BC) has been associated with an increased risk of ischemic heart disease (IHD). We examined the incidence of IHD in a large population-based cohort of women with BC.

Methods: The Breast Cancer DataBase Sweden (BCBaSe) includes all women diagnosed with BC from 1992 to 2012 $(n=60,217)$ and age-matched women without a history of $B C(n=300,791)$ in three Swedish health care regions. Information on comorbidity, educational level, and incidence of IHD was obtained through linkage with populationbased registries. The risk of IHD was estimated by Cox proportional hazard regression analyses and cumulative incidence by the Kaplan-Meier method.

Results: Women with $B C$ had a lower risk of $I H D$ compared to women without $B C$ with a hazard ratio (HR) of 0.91 ( $95 \% \mathrm{Cl}$ 0.88-0.95). When women with left-sided BC were compared to right-sided BC, an increased HR for IHD of 1.09 (95\% Cl 1.01-1.17) was seen. In women receiving RT, a HR of 1.18 (95\% Cl 1.06-1.31) was seen in left-sided compared to right-sided BC, and the HRs increased with more extensive lymph node involvement and with the addition of systemic therapy. The cumulative IHD incidence was increased in women receiving left-sided RT compared to right-sided RT, starting from the first years after RT and sustained with longer follow-up.

Conclusions: Women given RT for left-sided BC during 1992 to 2012 had an increased risk of IHD compared to women treated for right-sided $\mathrm{BC}$. These women were treated in the era of three-dimensional conformal RT (3DCRT), and the results emphasize the importance of further developing and implementing RT techniques that lower the cardiac doses, without compromising the beneficial effects of RT.
\end{abstract}

Keywords: Breast cancer, Radiotherapy, Survivorship, Ischemic heart disease, Long-term side effects

\section{Background}

Radiotherapy (RT) is associated with ischemic heart disease (IHD) due to incidental cardiac radiation exposure, and the IHD risk increases linearly with the radiation dose to the heart and coronary arteries without an apparent threshold dose [1-3]. Women irradiated for left-sided breast cancer $(\mathrm{BC})$ receive substantially higher doses to

\footnotetext{
* Correspondence: anakinwen@gmail.com

${ }^{1}$ Department of Surgical and Perioperative Sciences, Umeå University, Umeå, Sweden

${ }^{2}$ Department of Oncology, Sundsvall Hospital, Sundsvall, Sweden Full list of author information is available at the end of the article
}

the heart compared to women irradiated for right-sided $\mathrm{BC}$, and several trials show an increased IHD risk after RT in left-sided $\mathrm{BC}[1,4,5]$. A higher incidence of stenosis in the left anterior descending artery (LAD) has been reported after RT of left-sided compared to right-sided BC [5-8]. A large population-based study in women with BC treated from 1976 to 2006 showed an increase in acute myocardial infarction, angina pectoris, pericarditis, and valvular heart disease after left-sided RT compared to right-sided RT, with an enhanced risk in women diagnosed with IHD prior to BC [1]. The development of new RT techniques has decreased radiation doses to the heart. 
Even so, doses to the anterior part of the heart, especially the LAD, may still be high [9-11].

Many women with $\mathrm{BC}$ receive endocrine therapy and chemotherapy in addition to RT. Studies have shown a higher risk of cardiovascular disease in patients treated with aromatase inhibitors (AI) compared to tamoxifen, though this elevation in risk may be explained by a cardioprotective effect of tamoxifen [12, 13]. Cytotoxic agents, especially regimens including anthracyclines, are associated with an increased risk of congestive heart failure $[14,15]$. Anthracyclines combined with RT have been associated with a higher risk of heart disease in left-sided $\mathrm{BC}$ compared to RT alone, suggesting an additive effect $[16,17]$. The aim of the present study was to examine the risk of IHD in women with $\mathrm{BC}$ receiving $\mathrm{RT}$ and age-matched women without a history of $\mathrm{BC}$ in a large population-based cohort. The possible added risk from combining RT with endocrine therapy and chemotherapy was also studied.

\section{Methods \\ Study population}

Since the late 1970s, information on Swedish women with $\mathrm{BC}$ has been recorded in regional breast cancer registries. From 2008, all BC patients are registered in the National Quality Registry for Breast Cancer. For research purposes, the registries from three of Sweden's six health care regions (Stockholm, Uppsala-Örebro, and the Northern region) were merged to form the Breast Cancer DataBase Sweden (BCBaSe) cohort. All new cases of invasive BC in women from 1992 to 2012 were included. For each BC case, five women without a BC diagnosis, born in the same year as the index case, were added to form a comparison cohort. Women of the comparison cohort were allowed to become $\mathrm{BC}$ cases if diagnosed with $\mathrm{BC}$ during the study period. In the present study, all patients with early $B C$ were selected from the $\mathrm{BCBaSe}$ cohort, and patients with metastatic $\mathrm{BC}$ at diagnosis were excluded.

The study cohort was linked to the National Patient Register (NPR) and the Longitudinal Integration Database for Health Insurance and Labor Market Studies, using the unique personal identity numbers issued to all Swedish citizens. NPR is a validated registry containing records of all hospital discharges in Sweden since 1987 and also hospital-based outpatient care since 2001 [18]. The NPR contains information on the main diagnosis and up to eight secondary diagnoses. The Longitudinal Integration Database for Health Insurance and Labor Market Studies records data from the labor market and the educational and social sectors. This registry contains data on socioeconomic variables for all Swedish residents, such as marital status, income, place of employment, and the highest level of education [19].
Comorbidity was classified using the NPR according to the Charlson Comorbidity Index (CCI) in three comorbidity levels: CCI 0 (no comorbidity), CCI 1 (mild comorbidity), and CCI 2 (severe comorbidity) [20].

\section{Statistical methods}

IHD was defined according to the International Classification of Disease (ICD) 9th edition codes 410-414 or ICD-10 codes I20-I25, which include angina pectoris, acute myocardial infarction, complications due to myocardial infarction, and chronic IHD. To estimate the risk of IHD, Cox proportional hazard regression analysis was performed, using age as a timescale. The risk of IHD was examined by comparing women with $\mathrm{BC}$ to women without $\mathrm{BC}$ and by comparing women receiving left-sided RT to right-sided RT. The analyses were stratified for the type of surgery, RT, endocrine therapy, chemotherapy, and treatment with trastuzumab. The RT was also stratified based on the pathological lymph node status recorded in the breast cancer registries. According to the Regional $\mathrm{BC}$ treatment guidelines during the study period, women with no lymph node metastases were likely to receive RT to the breast or chest wall alone, and women with more than four positive lymph nodes were likely to receive loco-regional RT. The Regional BC treatment guidelines concerning RT in women with one to three positive lymph nodes varied over time and between the different regions included. This group most likely includes both women that received RT to the breast or chest wall alone and women that received loco-regional RT. Women with bilateral $\mathrm{BC}$ and women with unknown $\mathrm{BC}$ laterality were excluded in all analyses regarding the risk of IHD by laterality. Analyses concerning trastuzumab included only women with $\mathrm{BC}$ diagnosed from the year 2005.

The analyses were adjusted for the number of previous IHD events, time since last IHD event, non-cardiac comorbidity (CCI with IHD removed), and educational level (low, $<10$ years mandatory school; intermediate, $10-12$ years high school; and high, university or college). Women with $\mathrm{BC}$ were followed from the date of $\mathrm{BC}$ diagnosis (i.e., date of inclusion) to the date of the first IHD event registered in the NPR after inclusion, death, migration, or at the end of follow-up (31 December 2013), whichever came first. For women without $\mathrm{BC}$ in the comparison cohort who later were diagnosed with $\mathrm{BC}$, an additional end of follow-up was defined. Cumulative incidence of IHD was calculated by the Kaplan-Meier method separately for all women with BC; women with $\mathrm{BC}$ receiving left-sided $\mathrm{RT}$, right-sided $\mathrm{RT}$, or no RT; and women without BC. Analyses were performed using the statistical software R [21].

\section{Results}

\section{Study population}

The study population consisted of 60,217 women with $\mathrm{BC}$ and 300,791 women without a history of $\mathrm{BC}$, shown 
in Table 1. There were 28,903 women diagnosed with right-sided $\mathrm{BC}$ and 30,840 with left-sided $\mathrm{BC}$, while 474 women had bilateral $\mathrm{BC}$ or unknown $\mathrm{BC}$ laterality. The mean follow-up time was 8.1 years for women with $\mathrm{BC}$ and 9.0 years in women without $\mathrm{BC}$. The mean age at inclusion was 62.6 years. Women with $\mathrm{BC}$ had a higher educational level compared to women without $\mathrm{BC}$ diagnosis; $27.6 \%$ of the women with $\mathrm{BC}$ had an education longer than 12 years compared to $24.4 \%$ of the women without BC. No major differences were seen between women with $\mathrm{BC}$ and women without $\mathrm{BC}$ concerning CCI or a history of previous IHD events. The vast majority of women in the study population had no reported comorbidity. Previous IHD events were reported in 3.9\% of the women with $\mathrm{BC}$ and in $4.2 \%$ of the women without $\mathrm{BC}$. Women with $\mathrm{BC}$ who received no RT were

Table 1 Characteristics of the study population

\begin{tabular}{|c|c|c|c|c|}
\hline & BC all, $n(\%)$ & $\mathrm{BC}$ right, $n(\%)$ & BC left, $n(\%)$ & Women without $\mathrm{BC}, n(\%)$ \\
\hline No. of women & 60,217 & 28,903 & 30,840 & 300,791 \\
\hline FU time, yrs (SD) & $8.1(5.5)$ & $8.1(5.5)$ & $8.1(5.5)$ & $9.0(5.6)$ \\
\hline \multicolumn{5}{|l|}{ Health care region } \\
\hline Stockholm & $25,606(42.5)$ & $12,448(43.1)$ & $13,158(42.7)$ & $127,937(42.5)$ \\
\hline Uppsala/Örebro & $25,323(42.1)$ & $11,919(41.2)$ & $12,934(41.9)$ & $126,490(42.1)$ \\
\hline Northern region & $9288(15.4)$ & $4536(15.7)$ & $4748(15.4)$ & $46,364(15.4)$ \\
\hline \multicolumn{5}{|l|}{ Year of inclusion } \\
\hline 1992-1997 & $14,149(23.5)$ & $6578(22.8)$ & $7103(23.0)$ & $70,666(23.5)$ \\
\hline 1998-2002 & $14,285(23.7)$ & $6960(24.1)$ & $7320(23.7)$ & $71,362(23.7)$ \\
\hline $2003-2007$ & $14,960(24.8)$ & $7210(24.9)$ & $7749(25.1)$ & $74,740(24.8)$ \\
\hline 2008-2012 & $16,823(27.9)$ & $8155(28.2)$ & $8668(28.1)$ & $84,023(27.9)$ \\
\hline \multicolumn{5}{|l|}{ Age at inclusion } \\
\hline$<45 \mathrm{yrs}$ & $5770(9.6)$ & $2792(9.7)$ & $2935(9.5)$ & $28,826(9.6)$ \\
\hline $45-54$ yrs & $12,831(21.3)$ & $6199(21.4)$ & $6526(21.2)$ & $64,166(21.3)$ \\
\hline $55-64$ yrs & $16,054(26.7)$ & 7907 (27.4) & $8046(26.1)$ & $80,017(26.6)$ \\
\hline $65-74$ yrs & $13,928(23.1)$ & $6604(22.8)$ & $7203(23.4)$ & $69,691(23.2)$ \\
\hline $75-84$ yrs & $8101(13.5)$ & $3768(13.0)$ & $4257(13.8)$ & $40,461(13.5)$ \\
\hline $85 y r s+$ & $3533(5.9)$ & $1633(5.6)$ & $1873(6.1)$ & $17,630(5.9)$ \\
\hline \multicolumn{5}{|l|}{ Marital status } \\
\hline Married & $30,766(51.1)$ & $14,769(51.1)$ & $15,744(51.1)$ & $152,775(50.8)$ \\
\hline Not married & $29,451(48.9)$ & $14,134(48.9)$ & $15,096(48.9)$ & $148,016(49.2)$ \\
\hline \multicolumn{5}{|l|}{ Level of education } \\
\hline Low & $18,755(31.1)$ & 8844 (30.6) & $9684(31.4)$ & $100,623(33.5)$ \\
\hline Middle & $22,808(37.9)$ & $10,987(38.0)$ & $11,689(37.9)$ & $115,079(38.3)$ \\
\hline High & $16,626(27.6)$ & $8143(28.2)$ & $8420(27.3)$ & $73,246(24.4)$ \\
\hline Missing & $2028(3.4)$ & $929(3.2)$ & $1047(3.4)$ & $11,843(3.9)$ \\
\hline \multicolumn{5}{|l|}{$\mathrm{CCl}$} \\
\hline 0 & $50,825(84.4)$ & $24,447(84.6)$ & $25,985(84.3)$ & $255,689(85.0)$ \\
\hline 1 & $4788(8.0)$ & $2249(7.8)$ & $2483(8.1)$ & $24,597(8.2)$ \\
\hline 2 & $2879(4.8)$ & $1398(4.8)$ & $1470(4.8)$ & $13,350(4.4)$ \\
\hline $3+$ & $1725(2.9)$ & $809(2.8)$ & $902(2.9)$ & $7155(2.4)$ \\
\hline \multicolumn{5}{|c|}{ No. of previous IHD events } \\
\hline 0 & $57,877(96.1)$ & $27,775(96.1)$ & $29,641(96.1)$ & $288,263(95.8)$ \\
\hline 1 & $1421(2.4)$ & $679(2.3)$ & $732(2.4)$ & $7598(2.5)$ \\
\hline $2+$ & $919(1.5)$ & 449 (1.6) & $467(1.5)$ & $4930(1.6)$ \\
\hline
\end{tabular}

Characteristics of the study population by all women with breast cancer (BC), women with right-sided $B C$, women with left-sided $B C$, and women without $\mathrm{BC}$ diagnosis

No. Number, FU follow-up, yrs years, SD standard deviation, CCI Charlson Comorbidity Index 
older at the time of $\mathrm{BC}$ diagnosis compared to women with $\mathrm{BC}$ who received RT, 69 years vs. 60 years, respectively; had more comorbidities according to CCI ( $89 \%$ vs. $99 \%$ had $\mathrm{CCI}=0$ ); and were more frequently diagnosed with IHD (3.5\% vs. $1 \%)$ prior to $\mathrm{BC}$ diagnosis (data not shown in the table).

Patient characteristics for all women with $\mathrm{BC}$ and by laterality are shown in Table 2 . There were numerically more women with left-sided than right-sided BC, and the tumor characteristics were similar between these groups. A majority of the women with $\mathrm{BC}$ had a pathological tumor (T) stages 1 and $2(46.7 \%$ and $30.2 \%$, respectively). The pathological axillary lymph node stage was reported for $87.1 \%$ of the patients. Of those with reported data, $31.3 \%$ of the women were node-positive $(\mathrm{N} 1-3+/ \mathrm{N} 4+)$. Women with left-sided BC were less often operated with breast-conserving surgery (BCS), and there were also slightly fewer women with left-sided $\mathrm{BC}$ selected for RT compared to right-sided BC. No major differences regarding the use of endocrine treatment, chemotherapy, and trastuzumab were noticed between left-sided and right-sided BC.

\section{Risk of IHD for women with BC}

The risk of IHD for women with $\mathrm{BC}$ compared to women without $\mathrm{BC}$ is presented in Fig. 1, along with the number of IHD events, the number of women, and the person-years at risk. The risk of IHD was lower for women with $\mathrm{BC}$ compared to women without $\mathrm{BC}$ with a HR of 0.91 (95\% CI 0.88-0.95). Women with BC were stratified for different $\mathrm{BC}$ treatments and compared to women without BC. Women who received RT had a HR of 0.88 (95\% CI $0.83-0.92$ ), and women who received no RT of 0.95 (95\% CI $0.90-1.00)$ for IHD compared to women without BC. Analysis concerning surgery, other adjuvant therapies, and stratifications by pathological lymph node stages are displayed in Fig. 1.

\section{Risk of IHD for women with $B C$ by laterality}

The risk of IHD in women with left-sided BC compared to right-sided $\mathrm{BC}$ is shown in Fig. 2, along with the number of IHD events, the number of women, and the person-years at risk. There was a higher risk of IHD in left-sided BC compared to right-sided BC with a HR of 1.09 (95\% CI 1.01-1.17). For women not receiving RT, the risk of IHD in left-sided and right-sided $\mathrm{BC}$ was very similar (HR 1.00, 95\% CI 0.89-1.11) and without change when subdivided according to surgery. In women receiving RT, a higher risk of IHD in left-sided BC was seen, with a HR of 1.18 (95\% CI 1.06-1.31). The left/right HR for IHD was 1.25 (95\% CI 1.03-1.53) after mastectomy and RT and 1.13 (95\% CI 0.99-1.28) after BCS and RT. Subgroup analyses according to pathological lymph node stage showed an increasing risk of IHD in left-sided BC
Table 2 Patient characteristics for women with breast cancer by breast cancer laterality

\begin{tabular}{llll}
\hline BC characteristics & BC all, $n(\%)$ & BC right, $n(\%)$ & BC left, $n(\%)$ \\
\hline $\begin{array}{l}\text { No. of women } \\
\text { T status }\end{array}$ & $60,217(100.0)$ & $28,903(100.0)$ & $30,840(100.0)$ \\
T0 & $8616(14.3)$ & $4224(14.6)$ & $4391(14.2)$ \\
T1 & $28,100(46.7)$ & $13,527(46.8)$ & $14,289(46.3)$ \\
T2 & $18,185(30.2)$ & $8646(29.9)$ & $9390(30.4)$ \\
T3 & $2810(4.7)$ & $1329(4.6)$ & $1457(4.7)$ \\
T4 & $1165(1.9)$ & $560(1.9)$ & $605(2.0)$ \\
TX & $1341(2.2)$ & $617(2.1)$ & $708(2.3)$
\end{tabular}

$\mathrm{N}$ status

$\begin{array}{llll}\text { N0 } & 33,659(55.9) & 16,226(56.1) & 17,155(55.6) \\ \text { N1-3 } & 12,799(21.3) & 6154(21.3) & 6565(21.3) \\ \text { N4+ } & 5993(10.0) & 2823(9.8) & 3120(10.1) \\ \text { NX } & 7766(12.9) & 3700(12.8) & 4000(13.0)\end{array}$

ER status

$\begin{array}{llll}\text { ER+ } & 40,168(66.7) & 19,532(67.6) & 20,460(66.3) \\ \text { ER- } & 8666(14.4) & 4015(13.9) & 4587(14.9) \\ \text { Missing } & 11,383(18.9) & 5356(18.5) & 5793(18.8)\end{array}$

Missing $\quad 11,383(18.9) \quad 5356(18.5) \quad 5793(18.8)$

PR status

$\begin{array}{llll}\text { PR+ } & 33,335(55.4) & 16,216(56.1) & 16,955(55.0) \\ \text { PR- } & 14,873(24.7) & 7033(24.3) & 7765(25.2) \\ \quad \text { Missing } & 12,009(19.9) & 5654(19.6) & 6120(19.8) \\ \text { Surgery } & & & \\ \text { No surgery } & 2345(3.9) & 1115(3.9) & 1212(3.9) \\ \text { BCS } & 32,583(54.1) & 15,873(54.9) & 16,456(53.4) \\ \text { Mastectomy } & 23,917(39.7) & 11,268(39.0) & 12,453(40.4) \\ \text { Missing } & 1372(2.3) & 647(2.2) & 719(2.3)\end{array}$

RT stratified for pathological node stage

$\begin{array}{llll}\text { No RT } & 22,790(37.8) & 10,808(37.4) & 11,794(38.2) \\ \text { RT N0 } & 21,524(35.7) & 10,472(36.2) & 10,887(35.3) \\ \text { RT N1-3 } & 9088(15.1) & 4360(15.1) & 4658(15.1) \\ \text { RT N4+ } & 4679(7.8) & 2219(7.7) & 2423(7.9)\end{array}$

Endocrine therapy

\begin{tabular}{llll} 
Yes & $36,893(61.3)$ & $17,884(61.9)$ & $18,867(61.2)$ \\
No & $23,324(38.7)$ & $11,019(38.1)$ & $11,973(38.8)$ \\
$\begin{array}{l}\text { Chemotherapy } \\
\text { Yes }\end{array}$ & $16,552(27.5)$ & $7980(27.6)$ & $8528(27.7)$ \\
No & $43,665(72.5)$ & $20,923(72.4)$ & $22,312(72.3)$ \\
Trastuzumab & & & \\
Yes & $1943(7.5)$ & $919(7.3)$ & $1024(7.6)$ \\
No & $23,964(92.5)$ & $11,595(92.7)$ & $12,368(92.4)$ \\
\hline
\end{tabular}

Patient characteristics for women with breast cancer (BC) by BC laterality No. number, $T$ pathological tumor stage, $N$ pathological lymph node stage, $E R$ estrogen receptor, $P R$ progesterone receptor, $B C S$ breastconserving surgery, $R T$ radiotherapy

${ }^{a}$ Included women with BC registered from 2005 and later 


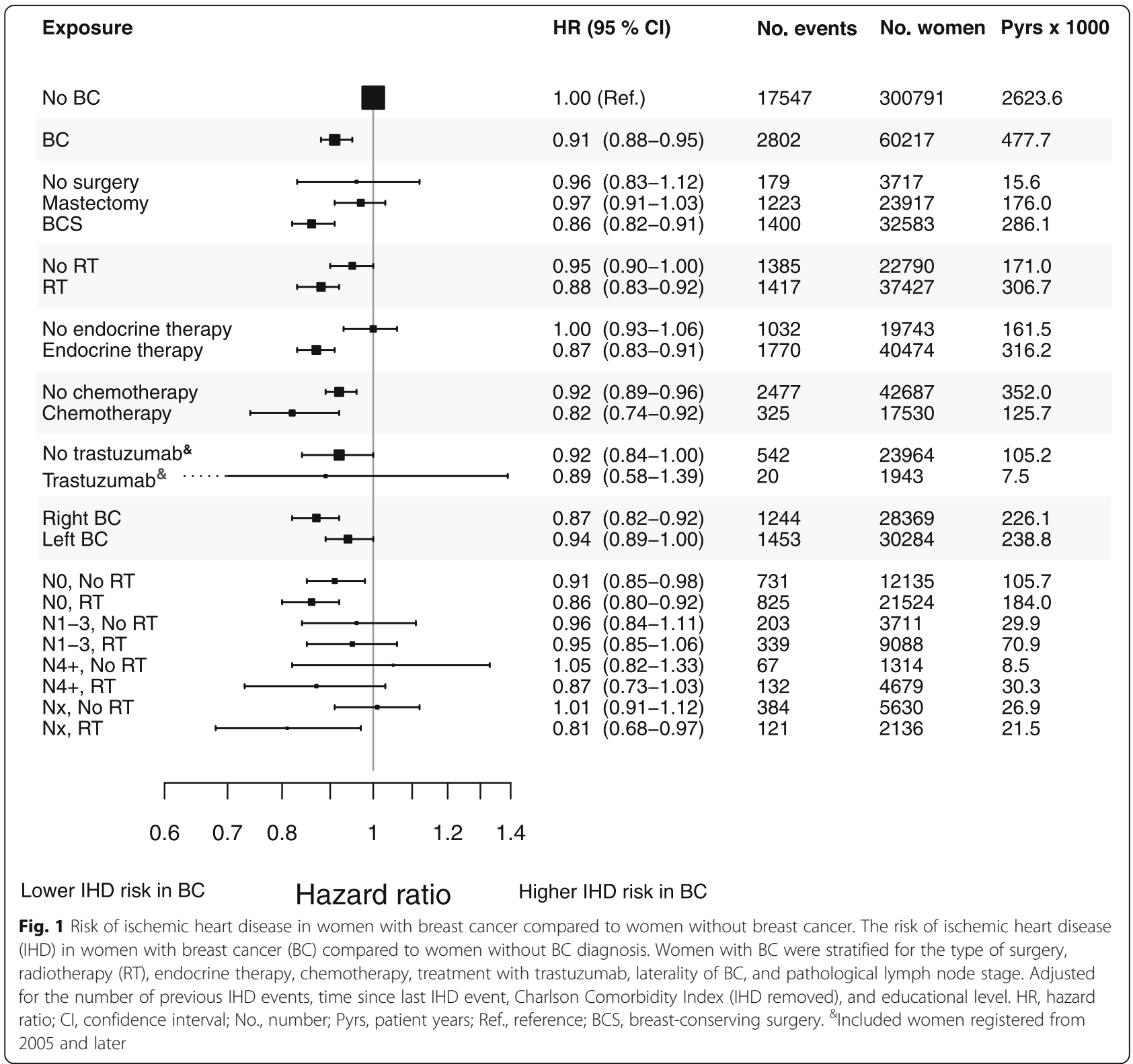

compared to right-sided $\mathrm{BC}$ in more advanced lymph node stages, with the largest IHD risk seen in patients with four or more metastatic lymph nodes, HR 1.46 (95\% CI 1.11-1.91). We found a higher left/right HR for IHD after RT in women receiving additional systemic adjuvant therapy; the HR was 1.24 (95\% CI 1.09-1.42), 1.28 (95\% CI $0.98-1.66$ ), and 1.35 (95\% CI $0.95-1.92$ ) for endocrine therapy, chemotherapy, and endocrine therapy and chemotherapy combined, respectively, compared to RT alone.

\section{Cumulative incidence of IHD}

The cumulative incidence of IHD during a 20-year follow-up for women without $\mathrm{BC}$, all women with $\mathrm{BC}$, women with $\mathrm{BC}$ not receiving $\mathrm{RT}$, women receiving leftsided and right-sided RT is visualized in Fig. 3. The cumulative incidence of IHD was highest in women with $\mathrm{BC}$ who received no RT, followed by women without $\mathrm{BC}$. In women who received RT, a higher cumulative incidence of IHD was seen in those receiving left-sided compared to right-sided RT. The difference between left- and right-sided RT was observed within the first years after RT, and it continued to increase with longer follow-up. The cumulative incidence rate of IHD at year 20 of follow-up was $11.9 \%$ for women without BC, $10.9 \%$ for all women with BC, $13.2 \%$ for women with BC not receiving $\mathrm{RT}, 10.3 \%$ for women receiving left-sided $\mathrm{RT}$, and $8.9 \%$ for women receiving right-sided RT. 


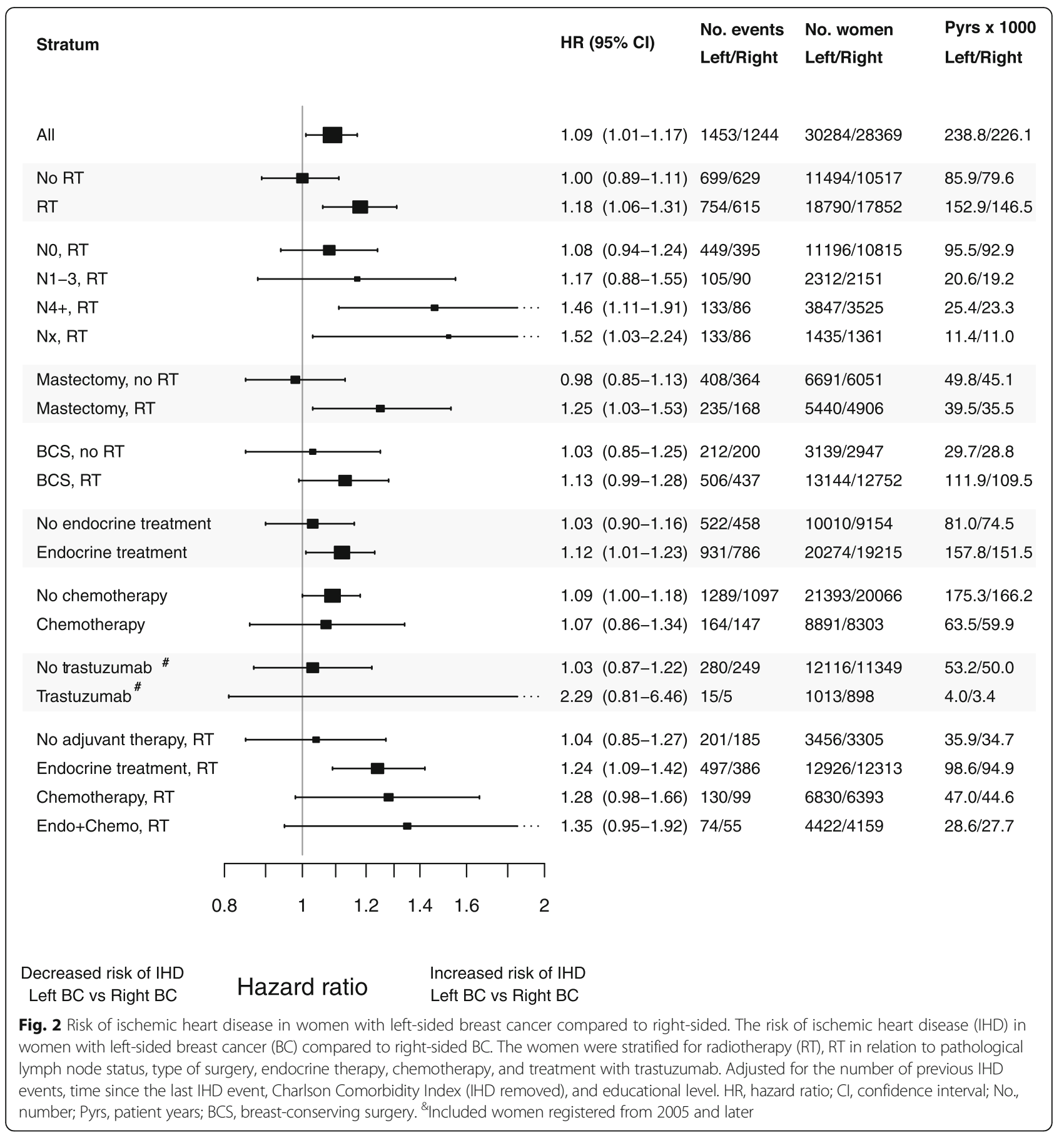

\section{Discussion}

The present study showed no difference in comorbidity between women with $\mathrm{BC}$ and women without $\mathrm{BC}$ at baseline, but at follow-up, a significantly lower risk of IHD was seen in women with BC compared to women without $\mathrm{BC}$. When women with $\mathrm{BC}$ receiving left-sided RT were compared to women receiving right-sided RT, a statistically significant $18 \%$ increased risk of IHD was seen. The HR's for IHD in women with left-sided RT compared to right-sided RT were even higher in women with more extensive lymph node metastasis, and when endocrine therapy and chemotherapy were added to RT. In concordance with the findings in the Cox regression, the cumulative incidence of IHD was higher for women receiving left-sided RT compared to right-sided RT. The difference was observed within the first years after RT, and it continued to increase with longer follow-up. 


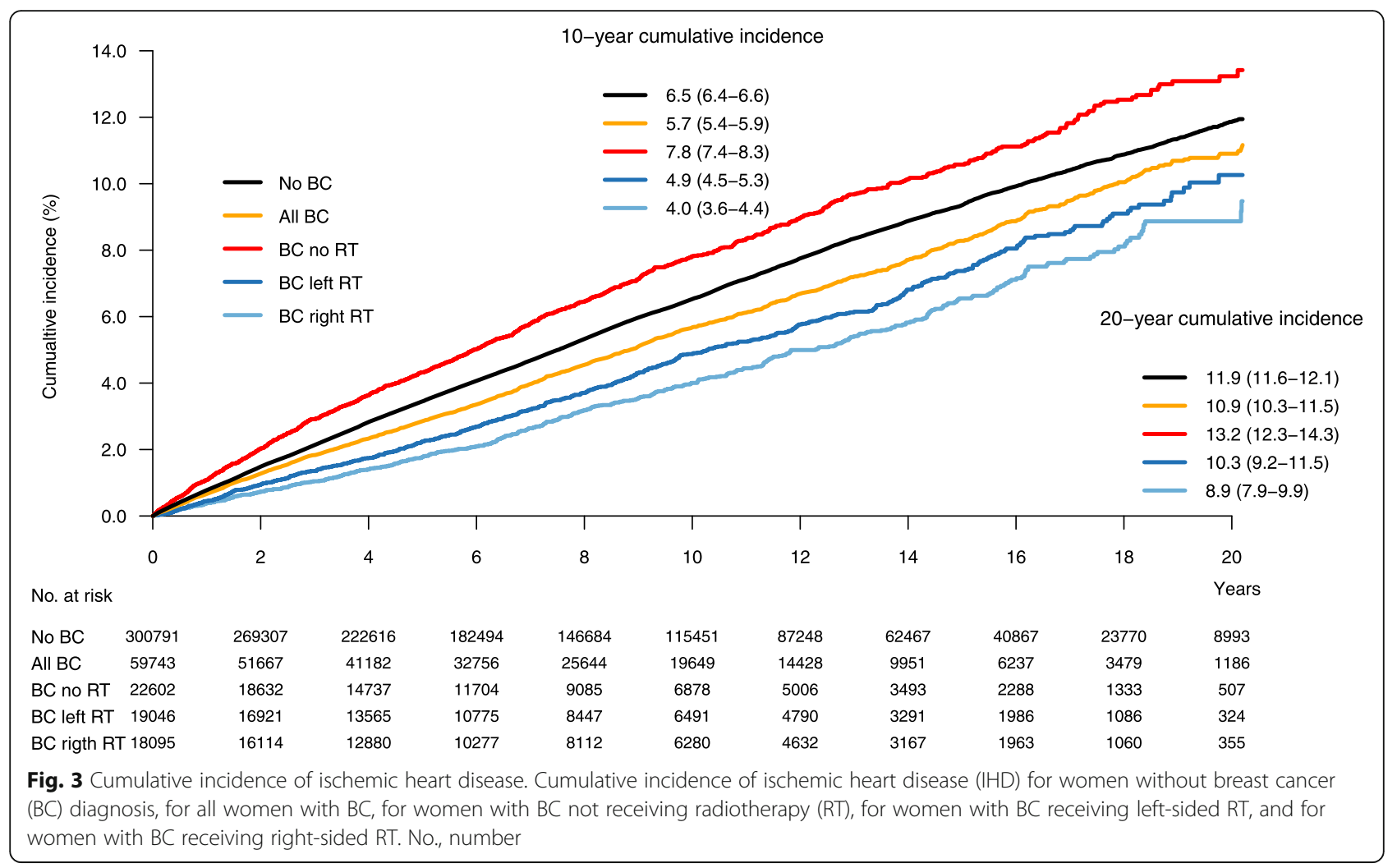

In line with other studies [22, 23], a lower risk of IHD was seen in women with $\mathrm{BC}$ compared to women without BC. The analysis was adjusted for educational level, $\mathrm{CCI}$, and the number of previous IHD events, but the findings may, however, be due to a confounding by a different distribution of lifestyle factors not captured by our adjustment. For example, some combinations of risk factors are negatively associated with IHD but positively with $\mathrm{BC}$ such as differences in child-bearing patterns, in the adoption of a healthy lifestyle and in adherence to mammography screening $[24,25]$.

The comparison to women without $\mathrm{BC}$ further shows that retrospective studies on cardiac toxicity will be biased if the background population is used as the control group.

The findings of lower risk of IHD in women with $\mathrm{BC}$ compared to women without BC were only observed in women recommended RT and other adjuvant therapies. Women with $\mathrm{BC}$ who received no RT had more comorbidities according to $\mathrm{CCI}$, including previous IHD events, and a selection of healthier women to active therapies has probably occurred. Retrospective comparisons between women that did receive treatments with women that did not would likely be severely biased.

Thus, the risk of IHD after RT in BC could not be assessed by comparing $\mathrm{BC}$ patients with age-matched women without $\mathrm{BC}$, nor by comparing women with $\mathrm{BC}$ who received $\mathrm{RT}$ to women with $\mathrm{BC}$ who did not.
Instead, we chose to analyze the risk of IHD by comparing left- and right-sided BC. The mean heart doses and doses to coronary arteries are considerably higher after left-sided RT $[6,9,26]$, but as cardiac structures also receive doses after right-sided RT, this comparison probably will underestimate the total added risk of IHD after $\mathrm{RT}$ in BC. In a recent systematic review of trials from 2010 to 2015, mean heart doses were 5.2 Gy and 3.7 Gy in left-sided and right-sided RT, respectively [11]. We recently showed considerably lower doses in a dosimetry study of $182 \mathrm{BC}$ patients selected from the same registry as the present study, although including only patients undergoing coronary angiography due to a cardiac event [27]. The median mean heart dose was $0.6 \mathrm{~Gy}$ (interquartile range (IQR) 0.4-1.0) after right-sided RT and 2.7 Gy (IQR 1.7-4.2) after left-sided RT. Moreover, the left/right RT dose difference was higher in the mid and distal LAD, where median mean doses were $3.6 \mathrm{~Gy}$ (IQR 2.4-6.2) and 26.7 Gy (IQR 7.0-41.0) after left-sided RT, and 0.5 Gy (IQR 0.1-0.8) and 0.3 Gy (IQR 0.1-0.8) after right-sided RT, respectively [27].

A higher risk of IHD in women with $\mathrm{BC}$ receiving leftsided RT compared to right-sided RT has also been shown in previous studies $[1,4,5,8]$. In this study, we show that this increase in risk persisted in women receiving RT for BC during 1992 to 2012. Most women were likely to receive three-dimensional conformal radiotherapy (3DCRT) given with tangential fields, but 
were treated before breathing adaption techniques, e.g., deep inspiratory breath-hold (DIBH), were implemented. The pattern of cumulative incidence further shows that the increase in the incidence of IHD in women with leftsided RT starts in the early years after RT and continues throughout follow-up.

An even higher risk of IHD was seen in women with more advanced pathological lymph node status. It is plausible that this group received more extensive RT including axillary and supraclavicular lymph nodes, and for some patients, the internal mammary chain (IMC), which is associated with higher cardiac radiation doses [6]. An increase in the risk of IHD was also seen when endocrine therapy and chemotherapy were added to RT in women with left-sided BC compared to right-sided BC. Anthracycline-based chemotherapy significantly increased the risk of cardiac death according to the 2005 EBCTCG meta-analysis, while tamoxifen use was associated with a lower cardiac death risk although the difference was not statistically significant $(p=0.06)$ [28]. A meta-analysis of published data from trials comparing aromatase inhibitors and tamoxifen reported a significant 30\% increase in cardiovascular morbidity with aromatase inhibitors [29]. Whether aromatase inhibitors increase cardiovascular disease compared to no endocrine treatment is still unclear. Two recent reports $[16$, 17] indicate that adjuvant anthracycline treatment may increase the risk of IHD after RT in breast cancer, especially when combined with RT to the IMC [17]. Since women who received adjuvant endocrine therapy and chemotherapy also had more advanced pathological lymph node status, the increased risk of IHD in women receiving both $\mathrm{RT}$ and systemic therapy has to be interpreted with caution.

Strengths of this study include the population-based setting, the large size of the cohort, and the linkage with other registries enabling adjustments for comorbidity and socioeconomic factors.

Several limitations need mentioning. Causes of death were not analyzed since in women with $\mathrm{BC}$, dying of $\mathrm{BC}$ is in this study design a competing risk to developing $\mathrm{IHD}$, and the analysis comparing women with BC to women without $\mathrm{BC}$ diagnosis may bias the risk of IHD in women with BC. Another limitation is that information concerning individual radiation doses and targets were not available. However, most women received a dose of 2 to 50 Gy over 5 weeks, according to Regional $\mathrm{BC}$ treatment guidelines throughout most of the study period. A few patients may have received hypofractionated RT schedules of 2.66 to $42.56 \mathrm{~Gy}$ or of 2.67 to $40.05 \mathrm{~Gy}$, as the guidelines allowed these schedules for selected cases from 2010. Most women with lymph node metastases also received RT to the axilla, the supraclavicular fossa, and in some cases the IMC, according to the
Regional BC treatment guidelines. A further limitation is that the median follow-up of 8.1 years for women with $\mathrm{BC}$ may be too short to draw conclusions regarding the risk of IHD for those women receiving RT during the latter part of the inclusion period. Previous studies have shown that the risk of radiation-induced IHD begins within a few years from RT and continues for a long period of time, with the highest risk seen between 10 and 14 years from the RT [2], and it is thus likely that the elevation in risk of IHD in women receiving leftsided RT in the present study will be even more pronounced at longer follow-up. One cannot exclude the possibility that women with a history of left-sided BC are observed with more caution due to awareness of side effects of the RT, which may lead to more frequent cardiovascular examinations and thus an increased opportunity to detect IHD. On the other hand, there were proportionally slightly fewer women with left-sided BC selected for RT compared to right-sided BC in this study, suggesting an initial selection of healthier women for left-sided RT.

\section{Conclusions}

We found an increase in the risk of IHD in women with left-sided BC compared to right-sided BC, given adjuvant RT during 1992 to 2012. Most of these women likely received 3DCRT given with tangential fields, but without DIBH techniques. The risk of radiation-induced IHD is expected to decrease when DIBH or other techniques that lower the cardiac and coronary doses are used. The results of this study emphasize the importance of fully implementing and further developing heartsparing techniques, without compromising the target coverage and the beneficial effects of RT. Even though the rate of IHD events after RT is relatively low, longterm side effects of adjuvant treatment have to be taken into consideration, to ensure health and quality of life for $\mathrm{BC}$ survivors.

\section{Abbreviations \\ RT: Radiotherapy; BC: Breast cancer; IHD: Ischemic heart disease; BCBaSe: Breast Cancer DataBase Sweden; Al: Aromatase inhibitors; NPR: National Patient Register; CCl: Charlson Comorbidity Index; ICD: International Classification of Disease; BCS: Breast-conserving surgery; HR: Hazard ratio; IMC: Internal mammary chain}

\section{Acknowledgements}

Not applicable.

Authors' contributions

AKW, CW, HG, CB, LH, GN, and MS performed the study design. AKW, CW, and $\mathrm{HG}$ performed all the statistical analyses and generated the figures. AKW was the major contributor in writing the manuscript. CW, HG, CB, LH, GN, IF, and MS edited the manuscript. All authors read and approved the manuscript.

\section{Funding}

This work was supported by grants from the Swedish Breast Cancer

Association (BRO), Visare Norr grant (grant no: 750491), the Department of 
Research and Development Västernorrland County Council (grant no: LVNFOU834391), and the Percy Falk Foundation for medical research. The funding body had no part in the design of the study; collection, analysis, and interpretation of the data; or writing the manuscript. Open access funding provided by Umea University.

\section{Availability of data and materials}

Further information and data sets are available from the corresponding author on reasonable request.

\section{Ethics approval and consent to participate}

All procedures performed in this study involving human participants were in accordance with the ethical standards of the regional committee of ethics in Stockholm, reference number 2013/1272-31/4 and with the 164 Helsinki Declaration and its later amendments or comparable ethical standards.

\section{Consent for publication}

Not applicable.

\section{Competing interests}

The authors declare that they have no competing interests.

\section{Author details}

'Department of Surgical and Perioperative Sciences, Umeå University, Umeå, Sweden. ${ }^{2}$ Department of Oncology, Sundsvall Hospital, Sundsvall, Sweden. ${ }^{3}$ Department of Surgery, Sundsvall Hospital, Sundsvall, Sweden. ${ }^{4}$ Regional Cancer Center, Uppsala University/Uppsala University Hospital, Uppsala, Sweden. ${ }^{5}$ Translational Oncology \& Urology Research (TOUR), School of Cancer and Pharmaceutical Sciences, King's College London, London, UK. ${ }^{6}$ Department of Breast-and Endocrine Surgery, Karolinska University Hospital, Stockholm, Sweden. ${ }^{7}$ Department of Molecular Medicine and Surgery, Karolinska Institutet, Stockholm, Sweden. ${ }^{8}$ Department of Oncology, Örebro University, University Hospital, Örebro, Sweden. ${ }^{9}$ Department of Surgical Sciences, Uppsala University, Uppsala, Sweden. ${ }^{10}$ Department of Immunology, Genetics and Pathology, Section of Experimental and Clinical Oncology, Uppsala University, University Hospital, Uppsala, Sweden. ${ }^{11}$ Department of Oncology, Gävle Hospital, Gävle, Sweden. ${ }^{12}$ Department of Oncology, Visby Hospital, Visby, Sweden.

Received: 30 July 2019 Accepted: 15 January 2020

Published online: 22 January 2020

\section{References}

1. McGale P, Darby SC, Hall P, Adolfsson J, Bengtsson NO, Bennet AM, et al. Incidence of heart disease in 35,000 women treated with radiotherapy for breast cancer in Denmark and Sweden. Radiother Oncol. 2011;100(2):16775 .

2. Darby SC, Ewertz M, McGale P, Bennet AM, Blom-Goldman U, Bronnum D, et al. Risk of ischemic heart disease in women after radiotherapy for breast cancer. N Engl J Med. 2013:368(11):987-98.

3. van den Bogaard VA, Ta BD, van der Schaaf A, Bouma AB, Middag AM, Bantema-Joppe EJ, et al. Validation and modification of a prediction model for acute cardiac events in patients with breast cancer treated with radiotherapy based on three-dimensional dose distributions to cardiac substructures. J Clin Oncol. 2017;35(11):1171-8.

4. Harris EE, Correa C, Hwang WT, Liao J, Litt HI, Ferrari VA, et al. Late cardiaC mortality and morbidity in early-stage breast cancer patients after breastconservation treatment. J Clin Oncol. 2006;24(25):4100-6.

5. Roychoudhuri R, Robinson D, Putcha V, Cuzick J, Darby S, Moller H. Increased cardiovascular mortality more than fifteen years after radiotherapy for breast cancer: a population-based study. BMC Cancer. 2007;7:9.

6. Taylor CW, Nisbet A, McGale P, Darby SC. Cardiac exposures in breast cancer radiotherapy: 1950s-1990s. Int J Radiat Oncol Biol Phys. 2007;69(5):1484-95.

7. Nilsson G, Holmberg L, Garmo H, Duvernoy O, Sjogren I, Lagerqvist B, et al. Distribution of coronary artery stenosis after radiation for breast cancer. J Clin Oncol. 2012:30(4):380-6.

8. Correa CR, Litt HI, Hwang WT, Ferrari VA, Solin LJ, Harris EE. Coronary artery findings after left-sided compared with right-sided radiation treatment for early-stage breast cancer. J Clin Oncol. 2007;25:3031-7.
9. Taylor CW, Povall JM, McGale P, Nisbet A, Dodwell D, Smith JT, et al. Cardiac dose from tangential breast cancer radiotherapy in the year 2006. Int J Radiat Oncol Biol Phys. 2007;25(21):3031-7.

10. Wennstig AK, Garmo H, Hallstrom P, Nystrom PW, Edlund P, Blomqvist C, et al. Inter-observer variation in delineating the coronary arteries as organs at risk. Radiother Oncol. 2017;122(1):72-8.

11. Taylor C, Correa C, Duane FK, Aznar MC, Anderson SJ, Bergh J, et al. Estimating the risks of breast cancer radiotherapy: evidence from modern radiation doses to the lungs and heart and from previous randomized trials. J Clin Oncol. 2017;35(15):1641-9.

12. Goldvaser H, Barnes TA, Seruga B, Cescon DW, Ocana A, Ribnikar D, et al. Toxicity of extended adjuvant therapy with aromatase inhibitors in early breast cancer: a systematic review and meta-analysis. J Natl Cancer Inst. 2018;110(1):31-9.

13. Khosrow-Khavar F, Filion KB, Al-Qurashi S, Torabi N, Bouganim N, Suissa S, et al. Cardiotoxicity of aromatase inhibitors and tamoxifen in postmenopausal women with breast cancer: a systematic review and metaanalysis of randomized controlled trials. Ann Oncol. 2017;28(3):487-96.

14. Sawyer DB. Anthracyclines and heart failure. N Engl J Med. 2013;368(12):1154-6.

15. Ryberg M, Nielsen D, Cortese G, Nielsen G, Skovsgaard T, Andersen PK. New insight into epirubicin cardiac toxicity: competing risks analysis of 1097 breast cancer patients. J Natl Cancer Inst. 2008;100(15):1058-67.

16. Rehammar JC, Jensen MB, McGale P, Lorenzen EL, Taylor C, Darby SC, et al. Risk of heart disease in relation to radiotherapy and chemotherapy with anthracyclines among 19,464 breast cancer patients in Denmark, 1977-2005. Radiother Oncol. 2017;123(2):299-305.

17. Boekel NB, Jacobse JN, Schaapveld M, Hooning MJ, Gietema JA, Duane FK, et al. Cardiovascular disease incidence after internal mammary chain irradiation and anthracycline-based chemotherapy for breast cancer. $\mathrm{Br} J$ Cancer. 2018;119(4):408-18.

18. Ludvigsson JF, Andersson E, Ekbom A, Feychting M, Kim JL, Reuterwall C, et al. External review and validation of the Swedish national inpatient register. BMC Public Health. 2011;11:450.

19. Statistiska centralbyrån: Longitudinell integrationsdatabas för sjukförsäkringsoch arbetsmarknadsstudier (LISA) http://www.scb.se/vara-tjanster/bestallamikrodata/vilka-mikrodata-finns/longitudinella-register/longitudinellintegrationsdatabas-for-sjukforsakrings\%2D\%2Doch-arbetsmarknadsstudierlisa/. Accessed July 11, 2019.

20. Charlson ME, Pompei $P$, Ales $K L$, Mackenzie CR. A new method of classifying prognostic comorbidity in longitudinal studies: development and validation. J Chronic Dis. 1987:40(5):373-83.

21. R Core Team: $R$ : a language and environment for statistical computing. $R$ Foundation for Statistical Computing, Vienna, Austria, 2018 https://www.Rproject.org/. Accessed July 11, 2019.

22. Jagsi R, Griffith KA, Koelling T, Roberts R, Pierce $L$ J. Rates of myocardial infarction and coronary artery disease and risk factors in patients treated with radiation therapy for early-stage breast cancer. Cancer. 2007:109(4):650-7.

23. Lamont EB, Christakis NA, Lauderdale DS. Favorable cardiac risk among elderly breast carcinoma survivors. Cancer. 2003:98(1):2-10.

24. Damiani G, Basso D, Acampora A, Bianchi CB, Silvestrini G, Frisicale EM, et al. The impact of level of education on adherence to breast and cervical cancer screening: evidence from a systematic review and meta-analysis. Prev Med. 2015;81:281-9.

25. Elshof LE, Schmidt MK, Rutgers EJT, van Leeuwen FE, Wesseling J, Schaapveld M. Cause-specific mortality in a population-based cohort of 9799 women treated for ductal carcinoma in situ. Ann Surg. 2018:267(5):952-8.

26. Nilsson G, Witt Nystrom P, Isacsson U, Garmo H, Duvernoy O, Sjogren I, et al. Radiation dose distribution in coronary arteries in breast cancer radiotherapy. Acta Oncol. 2016;55(8):959-63.

27. Wennstig AK, Garmo H, Isacsson U, Gagliardi G, Rintela N, Lagerqvist B, et al. The relationship between radiation doses to coronary arteries and location of coronary stenosis requiring intervention in breast cancer survivors. Radiat Oncol. 2019:14(1):40

28. Lancet. 2005;365(9472):Effects of chemotherapy and hormonal therapy for early breast cancer on recurrence and 15-year survival: an overview of the randomised trials, 1687-717.

29. Amir E, Seruga B, Niraula S, Carlsson L, Ocana A. Toxicity of adjuvant endocrine therapy in postmenopausal breast cancer patients: a systematic review and meta-analysis. J Natl Cancer Inst. 2011;103(17):1299-309.

\section{Publisher's Note}

Springer Nature remains neutral with regard to jurisdictional claims in published maps and institutional affiliations. 